\title{
Multiphysics Modeling of Nuclear Materials
}

Benjamin Spencer, Daniel Schwen, Jason Hales

July 2018

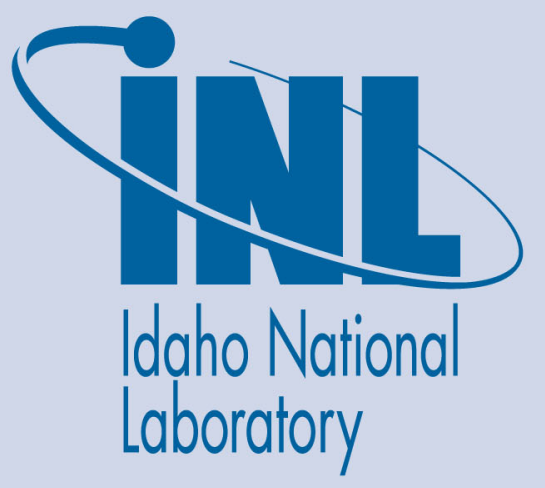

The INL is a U.S. Department of Energy National Laboratory operated by Battelle Energy Alliance 


\title{
Multiphysics Modeling of Nuclear Materials
}

\author{
Benjamin Spencer, Daniel Schwen, Jason Hales
}

July 2018

Idaho National Laboratory Idaho Falls, Idaho 83415

http://www.inl.gov

Prepared for the

U.S. Department of Energy

Under DOE Idaho Operations Office

Contract DE-AC07-05ID14517 


\section{Multiphysics Modeling of Nuclear Materials}

Benjamin Spencer, Daniel Schwen, and Jason Hales

This is published as: B. Spencer, D. Schwen, and J. Hales. Multiphysics Modeling of Nuclear Materials In W. Andreoni and S. Yip, eds. Handbook of Materials Modeling Springer International Publishing, Cham, 2018 DOI: 10.1007/978-3319-50257-1_131-1

The operating environment of nuclear reactors presents many unique challenges both for the materials comprising fuel elements (Olander 1976) and other structural components within and surrounding the reactor (Zinkle and Was 2013). These environmental challenges are due to the simultaneous exposure to conditions from multiple physical systems, including high temperatures, high radiation fluxes, aggressive chemical environments, and mechanical loading.

Numerical simulation has been used for multiple decades as a tool for understanding the behavior of various components in this environment. As these tools have been developed to increasing levels of sophistication, they have been used increasingly both for engineering analysis of components, as well as for simulating the processes of microstructure evolution, which lead to changes in the engineering properties of interest in the materials used in these components.

In some cases, the behavior of a single physics can be considered independently, simply because the response of one system does not change the conditions a component or material is subject to in other physical systems. For example, a component may be subjected to mechanical loading and thermal expansion due to elevated temperatures, but often, the mechanical response has a negligible effect on the thermal environment, so the temperature field can simply be imposed as a boundary condition to the mechanical model. However, there are also many scenarios in which the response to one physics has a strong effect on other physics. In the thermal/mechanical example, if the mechanical response of that component significantly changes its configuration, that could alter paths for heat transfer, and have a dramatic effect on the thermal field. In such a case, the simulation should account for two-way feedback between the models of these physical systems to accurately represent its response.

Benjamin Spencer

Fuel Modeling and Simulation Department, Idaho National Laboratory, Idaho Falls, ID 83415, email: benjamin. spencer@inl.gov 
There are many cases in which significant feedback occurs between physical systems within the nuclear reactor environment. These occur at length scales ranging from that of the full reactor system to the material level.

At the reactor scale, there is two-way feedback between the neutron transport and the thermal conditions within the nuclear fuel, which is volumetrically heated by the process of fissioning atoms under neutron flux. The nuclear cross-sections, or probabilities of interaction between a nucleus and an incident neutron, are dependent on the temperature. Because of the temperature dependence of the cross-sections, the fission rate is dependent on the fuel temperature.

Even if the fission rate is taken as a prescribed condition, computing the temperature in nuclear fuel is a complex multiphysics problem. In light water reactor (LWR) fuel, cylindrical uranium dioxide fuel pellets are stacked within long metallic fuel rods. Heat transfer from the fuel to the coolant outside the fuel rod is highly dependent on the size of the gap between the fuel and cladding, which can evolve due to the mechanical response of the system. Computing the thermal response of a fuel rod thus necessarily involves solving coupled equations of mechanics and heat conduction.

At the material scale, within ceramic nuclear fuel, exposure to irradiation and elevated temperatures causes significant changes in the grain structure of the fuel. Gaseous fission products diffuse through the solid material and form bubbles. These bubbles, along with larger pores, have a tendency to migrate up the temperature gradient. At high temperatures, grain growth occurs either in an equiaxial or columnar fashion, depending on the local conditions.

Similarly, phenomena affecting the material performance of the zirconium alloys used for cladding in LWRs are driven by multiple interacting physics. Exposure to coolant water causes atomic hydrogen to diffuse through the cladding. Some of this hydrogen precipitates in the form of hydrides, which can form, dissolve, reprecipitate, and change their orientation depending on the temperature and stress state. Under a constant temperature and far-field stress, the process of hydride formation is itself a multiphysics problem, as hydride formation induces local stresses due to the volumetric expansion that occurs during the phase change, and the process of that phase change is affected by the stress state.

Away from the fuel elements, the integrity of the structural elements comprising the reactor system is challenged by exposure to the reactor environment. These elements include core supporting structures, pressure vessels, control rods, piping, steam generators, and concrete structures such as biological shield walls and containment vessels.

Material evolution can be considered at many length and time scales. When considering phenomena of interest at the engineering scale, material behavior can often be homogenized, and represented as continuous behavior. When understanding of the processes of microstructure evolution is needed, simulations can be performed at the mesoscopic scale, and explicitly include features of the material structure, such as individual grains or constituents of a composite material. At lower scales, other modeling techniques can explicitly include features in crystal structures such as dislocations, voids, self-interstitial atoms, or other matrix defects. 
The discussion here is focused on modeling techniques for aspects of material behavior that vary spatially in a relatively continuous manner, so that the problem to be solved is to find spatially resolved field variables whose behavior is governed by partial differential equations (PDEs). The techniques for solving such problems are applicable at a wide variety of length scales, but are not applicable for modeling every aspect of multiphysics material response.

This chapter will first present a brief overview of the characteristics of the major physics affecting fuels and structural components in the nuclear reactor environment. This is followed by a discussion on the techniques used to numerically simulate these coupled systems. This includes both the discretization techniques and techniques for solving the resulting coupled systems of equations. Finally, several representative examples of coupled physics problems relevant to the performance of engineered systems and materials in a nuclear reactor environment are presented, along with details on the numerical simulation of those systems.

\section{Characteristics of Physics}

Multiple physical systems can potentially have a significant impact on the performance of nuclear reactor components and materials. These include mechanical deformation, heat transfer, species diffusion and reactions, phase change, fluid dynamics, and neutron transport. The characteristics of the physical systems that are typically most relevant to simulation of materials in nuclear reactors are summarized here.

\subsection{Solid Mechanics}

Materials and structures can experience mechanical deformation due to either external loading or intrinsically generated strains, such as those due to thermal expansion. The primary solution variable is the displacement vector $\boldsymbol{u}$, which varies spatially over the domain, and contains components for its values in the directions of the coordinate system employed.

Deformation is governed by stress equilibrium within the volume of the domain:

$$
\nabla \cdot \sigma+b=\mathbf{0}
$$

where $\boldsymbol{\sigma}$ is the stress tensor, and $\boldsymbol{b}$ is a vector of body forces (such as gravity loads). These tensors and vectors consist of components in the coordinate system of the model.

The strain tensor, $\varepsilon$, is related to the gradient of the displacement field through a kinematic relationship, shown here for the small-strain case:

$$
\varepsilon=\frac{1}{2}\left(\nabla \boldsymbol{u}+(\nabla \boldsymbol{u})^{T}\right)
$$


The treatment for finite strains is considerably more complex (Rashid 1993), and not covered here.

The stress is computed from the strain through a constitutive relationship, the details of which are material-specific. Under low stresses, many materials exhibit elastic behavior, in which the stress is linearly related to the applied strain through the elasticity tensor, $\boldsymbol{E}$ :

$$
\sigma=E: \varepsilon
$$

Intrinsically generated strains, (also referred to as eigenstrains) are subtracted from $\varepsilon$ before applying Equation 3. Under higher stresses, materials often exhibit nonlinear inelastic behavior in the form of creep or plasticity. This is usually represented by using an incremental form for stress computation, where stress is a function of the increment in the strain between the current and previous time steps (Simo and Hughes 1998).

The equations shown here are for the quasistatic case, where the deformation occurs slowly, or in other words, when the time derivatives of $\boldsymbol{u}$ are very small, so that inertial effects are assumed to be negligible. This is often the case for steady-state conditions in nuclear reactor components and materials. Additional terms for the effects of inertia and damping should be included in Equation 1 if these are important.

\subsection{Diffusion Processes}

Many physical systems are governed by diffusion processes. Heat transfer and species transport in solids are two such systems of interest in nuclear reactors. These are commonly represented by Fickian diffusion (Fick 1855), in which the change in local concentration of a given species, $\phi$ over time, $t$, is governed by the gradient of that concentration:

$$
\frac{\partial \phi}{\partial t}=D \nabla^{2} \phi
$$

where $D$ is the diffusion coefficient. This PDE can be solved for the concentration $\phi$, which is the primary solution variable. The flux vector $J$ of a system following this process is expressed as

$$
\boldsymbol{J}=-D \nabla \phi
$$

Heat transfer within a solid (in the absence of advection) is governed by Fickian diffusion. In this case, the concentration variable is the temperature, $T$. The energy balance equation governing heat transfer within a volume, including a source term due to an applied volumetric heating rate $\dot{Q}$, is expressed as:

$$
\rho C_{p} \frac{\partial T}{\partial t}=k \nabla^{2} T+\dot{Q}
$$

where $\rho$ is the density, $C_{p}$ is the specific heat at constant pressure, and $k$ is the thermal conductivity. The coefficients $C_{p}$ and $k$ can depend on temperature, making this a nonlinear equation. 
Many diffusion systems of interest are also affected by the Soret effect (Rahman and Saghir 2014), where the concentration of a species is a function of both the concentration gradient and the temperature. An example of such a system in a nuclear environment is hydrogen concentration in LWR fuel cladding (Stafford 2015). Heat and moisture transport in concrete are affected by both the Soret effect and its counterpart, the Dufour effect, in which temperature is affected by the concentration gradient (Bažant et al 1981).

\subsection{Phase Field Microstructure}

In ideal solutions, the diffusive driving force is represented by the concentration gradient, and species concentrations can be computed using diffusion models as described in the previous section. However, in systems other than ideal solutions, where the chemical potential is not a linear function of the concentration, the diffusive driving force is given by the gradient of the chemical potential rather than the concentration gradient. The modeling of species transport and phase changes on the mesoscale in these systems thus requires knowledge of the system's thermodynamic potential and its kinetic coefficients.

The phase-field method is a well-established tool for simulating the co-evolution of microstructure and physical properties at the mesoscale. In the phase-field method, the microstructure is described by a system of continuous variables, also called order parameters. Microstructure interfaces are approximated using a finite width, and the order parameters vary smoothly over those interfaces. The evolution of nonconserved order parameters $\eta_{j}$ (e.g. phase regions and grains) is governed by the Allen-Cahn (Allen and Cahn 1972) equation (7), and the evolution of conserved order parameters $c_{i}$ (e.g. concentrations) is governed by the Cahn-Hilliard (Cahn and Hilliard 1958) equation (8):

$$
\begin{aligned}
\frac{\partial \eta_{j}}{\partial t} & =-L_{j} \frac{\delta F}{\delta \eta_{j}}, & j & =1, \ldots, N_{\eta} \\
\frac{\partial c_{i}}{\partial t} & =\nabla \cdot\left(M_{i} \nabla \frac{\delta F}{\delta c_{i}}\right), & i & =1, \ldots, N_{c}
\end{aligned}
$$

Here, $F$ is the total free energy of the system, which can be formulated as a volume integral

$$
F=\int_{\Omega}\left(f_{\text {loc }}+f_{\text {gr }}+E_{\mathrm{d}}\right) \mathrm{d} V,
$$

where $\Omega$ is the simulation domain,

$$
f_{\mathrm{loc}} \equiv f_{\mathrm{loc}}\left(\eta_{1}, \eta_{2}, \ldots, c_{1}, c_{2}, \ldots\right)
$$

is the local free energy density, and 


$$
f_{\mathrm{gr}} \equiv f_{\mathrm{gr}}\left(\nabla \eta_{1}, \nabla \eta_{2}, \ldots, \nabla c_{1}, \nabla c_{2}, \ldots\right)
$$

is the gradient energy contribution.

In isolated systems, the evolution of these variables leads to a monotonically decreasing free energy as a function of time. The phase-field method has been used to model a large range of physical phenomena, including solidification, phase transformation, and grain growth.

\section{Discretization Techniques}

The behavior of physical systems is continuous in both space and time. To make the solution amenable to numerical solution, it is necessary to approximate the continuous response of the actual system using a finite number of variables. This process, known as discretization, allows the model to be solved using an algebraic system of equations. Discretization is used both in space and time - spatial discretization is used to describe the spatial variation of field quantities, while time discretization is used to solve for the system's response at a fixed number of times.

\subsection{Finite Element Method}

The finite element method (FEM) is widely used as a discretization technique for solving a variety of PDEs (Zienkiewicz et al 2013). In FEM, the solution domain is represented by a mesh, which is a collection of connected primitive geometric elements. Associated with these elements is a set of nodes, which are located at the vertices of elements, and can also be located on edges or faces, or within the volume of elements. Continuity of the solution between neighboring elements is enforced when those elements share common nodes.

FEM is used to solve a discretized form of the governing PDEs, which are enforced in a weak, or integral, form at the finite element degrees of freedom. The solution is locally interpolated over individual elements using a set of shape functions, which are associated with the degrees of freedom in the discrete system. A number of different types of shape functions can be used. Lagrangian shape functions are among the most commonly used and interpolate between degrees of freedom directly associated with the nodes of a given element.

The solution field, denoted here as $u(\boldsymbol{x})$, is interpolated at any point $\boldsymbol{x}$ within the domain by a set of interpolation, or shape functions $\phi_{i}$. Lagrangian shape functions are associated with the finite element nodes, so that for node $i, \phi_{i}=1$, and at all other nodes, $\phi_{i}=0$. At any location within an element, the sum of the shape functions is 1 . The solution and its gradient can be expressed at any location in the domain as a function of the shape functions and their gradients: 


$$
\begin{aligned}
u(\boldsymbol{x}) & =\sum_{i} \phi_{i}(\boldsymbol{x}) u_{i} \\
\nabla u(\boldsymbol{x}) & =\sum_{i} \nabla \phi_{i}(\boldsymbol{x}) u_{i}
\end{aligned}
$$

where $u_{i}$ are the values of $u$ at the nodes of the element containing point $\boldsymbol{x}$.

To solve a PDE using FEM, it must be cast in a variational, or weak form. This involves pre-multiplying each term of the PDE by a test function, and expressing the PDE as a domain integral. In the Galerkin method, these test functions correspond to the shape functions. The divergence theorem is then applied to integrate terms with divergence operators into two separate terms involving a volume and a surface integral, and which can be represented using gradients of the shape functions.

Finally, the weak form of the governing equation is integrated numerically. The integrals over the domain are performed by taking a summation of the integrals over individual elements, and the individual elements are integrated using numerical quadrature, typically Gaussian quadrature. An arbitrary function $f(\boldsymbol{x})$ is integrated numerically over the domain of an element $\Omega_{e}$ as:

$$
\int_{\Omega_{e}} f(\boldsymbol{x}) \approx \sum_{q} f\left(\boldsymbol{x}_{q}\right) w_{q}
$$

where $q$ are the quadrature points, $\boldsymbol{x}_{q}$ are the coordinates of the quadrature points, and $w_{q}$ are the weights of the quadrature points for an element.

An important side-effect of the use of quadrature is that while solution variables can be interpolated anywhere in the domain, coefficients in the PDE that are computed at quadrature points are only available at those locations, and extrapolation to other locations is somewhat problematic.

One of the advantages of FEM is that it is very flexible. It has become widely used for solving PDEs associated with a variety of physics. Finite element meshes can be constructed to conform to the shapes of very general domain geometries, so it can be used for modeling a variety of engineering components. The flexibility in mesh generation also readily permits local refinement in regions where increased fidelity is needed.

\subsection{Other Methods}

FEM is the most widely adopted technique for multiphysics PDE solvers, and is the basis for the methods discussed here. However, a number of other PDE solution techniques can be used for solving multiphysics problems, and a few of them are briefly discussed here. 
Finite Difference Method

The finite difference method is a technique for discretizing PDEs in either space or time, and was developed independently of FEM. FDM also uses a mesh to represent the solution domain, but the mesh must be topologically regular. It can be adjusted to fit domains with curved surfaces, but it does not have nearly the flexibility that FEM meshes do to conform to parts with complex geometry.

FDM is much simpler than FEM. FDM approximates the spatial derivatives of solution field variables by computing the differences between values of those variables on opposing sides of a given node in each coordinate direction. It does not rely on quadrature or interpolation functions in the way that FEM does.

FDM can be very numerically efficient, and although it is limited in the geometries that it can represent, it is very well-suited to material-scale modeling where the solution domain commonly consists of periodic representative volume elements.

\section{Spectral Methods}

Rather than discretizing the domain using degrees of freedom tied to specific material points, as is done in the FEM or FDM, the spatial variation of the solution field can be represented using a Fourier series expansion (Chen and Shen 1998). This technique is widely used in phase field simulations of periodic representative volume elements and is very efficient.

\section{Peridynamics}

Peridynamics is another method for solving governing equations. Peridynamics is a nonlocal meshfree method that solves integral, rather than differential equations (Silling 2000). Its development was driven by a need to better handle fracture in solid mechanics simulations. Using PDEs, along with the solution methods developed to solve PDEs, to represent mechanical deformation is problematic when material fractures, because the response is no longer continuous. Peridynamics overcomes some of the shortcomings of FEM in this area by allowing connections between material points to naturally break. It has since been generalized to other physics, and has been applied to coupled physics models, including nuclear fuel applications (Oterkus and Madenci 2017).

\section{Solution Techniques}

These discretization methods for PDEs all result in the representation of the system as a set of coupled algebraic equations. The integration of these equations in time can be done using explicit methods, in which no equation solution is needed, or implicit 
methods, which require the solution of a system of linear or nonlinear equations at each time step. Many of the materials challenges in nuclear reactors involve slowly evolving processes, which are more amenable to solution using implicit techniques, so those are the focus of the present discussion.

Typically, Newton's method or a variant thereof is used to solve the nonlinear system of equations for a given physics. The goal of the nonlinear solver is to obtain the solution vector $\boldsymbol{x}$ for which the associated residual $\boldsymbol{r}$ is zero:

$$
r(x)=0
$$

The Jacobian matrix $\boldsymbol{J}$ is:

$$
\boldsymbol{J}(\boldsymbol{x})=\frac{\partial \boldsymbol{r}(\boldsymbol{x})}{\partial \boldsymbol{x}} .
$$

In Newton's method, the following procedure is iteratively repeated to update the solution until Equation 15 is met within an acceptable tolerance:

$$
\begin{aligned}
& \text { Compute } \boldsymbol{J}\left(\boldsymbol{x}_{k}\right), \boldsymbol{r}\left(\boldsymbol{x}_{k}\right) \\
& \text { Solve } \boldsymbol{J}\left(\boldsymbol{x}_{k}\right) \boldsymbol{s}=-\boldsymbol{r}\left(\boldsymbol{x}_{k}\right) \text { for } \boldsymbol{s} \\
& \boldsymbol{x}_{k+1}=\boldsymbol{x}_{k}+\boldsymbol{s}
\end{aligned}
$$

where $s$ is the iterative update to the solution vector, and $k$ is the nonlinear iteration index.

Solving coupled physics systems introduces many mathematical and software engineering challenges that are not encountered in solving single physics equations. For multiphysics simulations, the complexity of solving the system is increased significantly because the solution vector $\boldsymbol{x}$ contains the unknown degrees of freedom for all of the physical systems modeled. To solve the system for all those physics using Newton's method requires forming the full Jacobian matrix for that coupled system. This can be quite challenging, because computing the terms related to the interactions between the physics can be very complex.

To avoid this difficulty, it is common to solve coupled physics problems in a loosely coupled fashion, where the systems of equations for the individual physics are solved independently. The results from the solutions of individual physics models are sequentially transferred to the other models. Fixed point iterations can be performed to iterate between these models until they reach a converged solution. Alternatively, if there is weak coupling between the systems, it may be acceptable to simply transfer the solutions from the other physics from the previous time step, and skip these iterations entirely.

This loose coupling approach allows for the individual physics models to be based on completely different solution and discretization schemes. The individual simulation codes and discretizations can be optimized for specific physics, and all that is required is a means to transfer results between the models. This approach is widely used in practice to couple physics codes that were developed independently.

For coupled physical systems with significant feedback, however, loose coupling approaches often suffer from decreased performance relative to tight coupling. A 
study performed by Novascone et al (2015) compared the performance of loose and tight coupling approaches for thermal/mechanical systems. It found that for weakly coupled systems, there were not significant benefits to using a tightly coupled solution strategy, but using tight coupling significantly improved performance for problems with strong two-way feedback between the physics.

The Jacobian-free Newton Krylov (JFNK) method, which is a variant of the Newton method that does not explicitly require the Jacobian (Knoll and Keyes 2004), has great utility for solving multiphysics problems as a single coupled system of equations since it alleviates some of the burden of forming the coupling terms in the Jacobian matrix. This algorithm employs a Krylov iterative method, such as GMRES (Saad and Schultz 1986), to compute the Newton iterative update in Equation 18:

$$
\boldsymbol{s}=a_{0} \boldsymbol{r}_{0}+a_{1} \boldsymbol{J} \boldsymbol{r}_{0}+a_{2} \boldsymbol{J}^{2} \boldsymbol{r}_{0}+\cdots+a_{l} \boldsymbol{J}^{l} \boldsymbol{r}_{0}
$$

where $\boldsymbol{r}_{0}$ is the initial residual, and $a_{i}$ are the series of coefficients computed in the individual Krylov iterations.

The Krylov algorithm only requires the action of the Jacobian on a vector $\boldsymbol{v}$, which can be approximated with the finite difference expression:

$$
\boldsymbol{J}\left(\boldsymbol{x}_{k}\right) \boldsymbol{v} \approx \frac{\boldsymbol{r}\left(\boldsymbol{x}_{k}+\epsilon \boldsymbol{v}\right)-\boldsymbol{r}\left(\boldsymbol{x}_{k}\right)}{\epsilon} .
$$

where $\epsilon$ is a small number that reflects a perturbation from the last iterative solution. As long as the Krylov iterations in Equation 20 are sufficiently converged, this technique has the same nonlinear convergence characteristics as Newton's method. There are two primary advantages to not explicitly forming a Jacobian: it can require significant computational resources to store and solve using the Jacobian, and it can be challenging to compute the off-diagonal coupling terms in the Jacobian.

Although the full Jacobian matrix is not strictly required in the JFNK algorithm, The Krylov iterations converge much more rapidly if the system is well-conditioned, so preconditioning is typically used with this approach. Using the exact Jacobian matrix for preconditioning will yield the best convergence rates for the Krylov iterations, but an approximation of it often performs quite well.

In multiphysics simulations, it is often fairly straightforward to compute the residual for physics as a function of the state of another physics, but computing the derivatives with respect to that other physics can be much more difficult. Hence the JFNK algorithm can be very attractive in that those derivatives are not strictly required. A working initial capability can be rapidly developed without those derivatives, and then they can be developed later as needed to optimize the algorithm.

An alternative approach to deal with the complexity of forming the Jacobian is to use automatic differentiation (AD) techniques to automatically compute the derivatives needed for the Jacobian from the code that computes the residual (Pawlowski et al 2012a). The residual evaluation code can be broken down into basic mathematical operations with known rules for computing derivatives. AD can be used to compute an exact Jacobian matrix for an entire equation system, which can be applied very effectively to obtain the solution. The main drawback to $\mathrm{AD}$ is that it requires 
sophisticated software engineering. For the derivatives to be evaluable from the code that computes residuals typically requires quite invasive changes to be made to that code.

A number of commercial and open source codes and frameworks supporting multiphysics simulations are available, based on a variety of designs. A few notable examples, all based on FEM, are mentioned here.

Many commercial codes such as Abaqus (Systemes 2016), which started out as single-physics solid mechanics codes, now support coupling with multiple physics. COMSOL (COMSOL, Inc 2017) is a commercial product specifically targeted at multiphysics simulation, and which permits adding user-defined physics. It has been used as the basis of a nuclear fuel simulation tool (Liu et al 2016).

Multiple frameworks have been developed with an explicit goal of being the basis for developing multiphysics simulation capabilities, with very different philosophies. A few examples of such frameworks are mentioned here.

The SIERRA framework (Stewart and Edwards 2004) is an example of a software framework based on the use of loose coupling to handle the solution of multiphysics models. It does this by providing common finite element data structures and services to transfer solutions between applications, which solve their individual physics equations independently.

Other frameworks solve multiphysics models using a single coupled system of equations. One such framework is MOOSE (Gaston et al 2009), which provides a pluggable set of interfaces to define individual physics models, and manages the FEM assembly of the residuals from those models, using preconditioned JFNK and other techniques to solve the physics models as a single, tightly coupled system. MOOSE is notable in the context of this chapter because its development was motivated specifically for the solution of simulation problems in nuclear energy. Multiple applications based on that framework have been developed for various aspects of nuclear energy simulations, and are used as the basis for the results shown here. Other open-source frameworks that solve a single system of equations are Trilinos (Pawlowski et al 2012b), FEniCS (Logg et al 2012), and Life (Prud'homme 2006). These also provide pluggable interfaces to define physics models and solve the coupled physics equations in a single tightly-coupled system, but they focus on using $\mathrm{AD}$ techniques to automate the construction of the Jacobian matrix.

\section{Applications}

\subsection{Fuel Performance}

A wide variety of nuclear reactors, with specialized nuclear fuels, have been designed or proposed. These include light water reactors (LWRs), research reactors employing plate fuel, fast reactors, high temperature gas reactors, and others. The discussion here focuses on the fuel performance of $\mathrm{UO}_{2}$ /zirconium alloy fuel rods 
for LWRs. These rods consist of hundreds of $\mathrm{UO}_{2}$ pellets atop one another encased in a zirconium alloy cladding with a gas-filled plenum at the top of the cladding.

The fundamental quantity of interest in LWR fuel performance analysis is the fuel center-line temperature. This quantity is critical due to its affect on neutronics and other fuel performance behaviors.

Considering Equation 6, for steady operation the important parameters are the volumetric heating rate and the thermal conductivity. The volumetric heating rate directly depends on the fission rate, which is given as in input for fuel performance analysis. The thermal conductivity of the ceramic fuel is quite low, whereas the thermal conductivity of the zirconium alloy cladding is fairly high given that the cladding is a metal. Thus the more important quantity is the fuel thermal conductivity.

Fuel thermal conductivity is temperature dependent (Fink 2000) as well as dependent on irradiation effects (Lucuta et al 1996). This being the case, the thermal conductivity of the fuel varies across the fuel pellet due to the temperature gradient in the pellet and also varies in time. The heat transfer equation is therefore nonlinear.

The heat in the fuel passes through the gap between the fuel and the cladding before passing through the cladding and then to the coolant. The gap between the fuel and cladding is filled with helium gas when the rod is fabricated. The ease at which heat passes through the gap is governed by the size of the gap, the thermal conductivity of the gas in the gap, pressure due to mechanical contact, if any, between the fuel and the cladding, and radiative heat transfer effects. The size of the gap depends on the initial geometry and the deformation of the fuel and cladding, which ties the mechanical response of the fuel rod to the thermal response. In addition, one product of nuclear fission is gas (primarily xenon and krypton) that will accumulate in the gap over time. These gaseous fission products affect the internal pressure of the fuel rod (the mechanical response) and the thermal conductivity of the gas mixture (the thermal response).

Conductance across the gap due to the gas follows Equation 22

$$
h_{g a p}=\frac{k_{g}\left(T_{g}\right)}{r_{c}-r_{f}+d}
$$

where $h_{\text {gap }}$ is the gap conductance, $k_{g}$ is the gas thermal conductivity, $T_{g}$ is the temperature of the gas, $r_{c}$ and $r_{f}$ are the radial positions of the cladding and fuel, respectively, and $d$ is an addition distance that accounts for roughness of the surfaces, for example. The need to calculate the distance between the cladding and the fuel clearly shows a dependence on the mechanical solution.

Other important fuel behaviors include densification, swelling, cracking, and creep. Early in life in the reactor, pellets densify and shrink slightly. Fuel swelling happens continuously under irradiation and is of two types. The first is solid fission product swelling and occurs in a linear relationship with irradiation. The second is due to gaseous fission products and is much more difficult to predict. Gaseous swelling depends on the diffusion of gas atoms and bubbles within grains, the accumulation and interconnection of gas bubbles at grain boundaries, and the eventual release of fission gas to the gap and plenum. 
When a fuel rod is initially brought to power in the reactor, the temperature gradient causes the pellet to crack due to thermal stresses. This cracking relieves stress and allows the pellet to move outward, slightly, in the radial direction. (This also affects temperature predictions because of the effect on the gap size.) Cracking also greatly influences the creep of the fuel pellet due to its effect on the stress state.

Thermal and irradiation creep, along with irradiation growth, of the zirconium alloy cladding are other behaviors that must be modeled. The coolant pressure in LWRs engages creep mechanisms in cladding, causing the cladding to move inward toward the pellet, affecting internal rod pressure, gap size, and, once again, temperature.

The effects of several sources of eigenstrains must be accounted for in the computation of the stress. For an example of how this is done, the elastic stress computation takes these into account as:

$$
\sigma=E\left(\varepsilon-\varepsilon_{T}-\varepsilon_{s w}-\varepsilon_{d}-\ldots\right)
$$

where $\sigma$ is the stress, $E$ is the elasticity tensor, $\varepsilon$ is the total mechanical strain, $\varepsilon_{T}$ is the thermal strain, $\varepsilon_{s w}$ is the strain due to swelling, and $\varepsilon_{d}$ is the swelling due to densification. Given that stress is dependent on the thermal strain, the displacement solution has a direct dependence on the temperature solution.

Other behaviors of interest in fuel performance modeling include the shift of power toward the outer rim of the fuel pellet, the thermal boundary condition on the exterior of the cladding, corrosion of the cladding, thermal expansion, the evolving internal rod pressure, mechanical contact between the fuel and cladding, and mass diffusion.

Most, though not all, fuel performance codes resolve the thermal and mechanical physics with a loose coupling approach. In this approach, the temperature at the grid points is computed based on a fixed set of displacements and then the displacements and stresses are computed based on those temperatures. The new stress state and displacements are then used to update the temperature, and the process is repeated until a satisfactory condition is met. It is worth noting that this approach can be nested. For example, the displacements may be computed using a fixed internal rod pressure. With new displacements, the internal rod pressure can be recomputed, and the two steps repeated to reach a final converged displacement solution.

Fuel performance codes are used to compute fuel center-line temperatures when comparing against experimental data, for postulated conditions, or when coupling with other (e.g. neutronics) codes. There is particular interest in using fuel performance codes to simulate abnormal, or accident, conditions. Because the fuel properties and behavior are outside normal regimes and can exhibit highly nonlinear behavior, this can be particularly challenging for fuel performance analysis.

For example, in a loss of coolant accident, the supply of coolant to the reactor is interrupted. This results in increased fuel rod and coolant temperatures. If coolant flow is not restored, the coolant will change to steam, and the fuel rod temperatures will continue to rise. A lack of external pressure in conjunction with increased tem- 
peratures can cause the cladding to creep outward. This outward motion may result in a ballooned section of the fuel rod and in cladding burst.

This situation requires more advanced thermal boundary conditions for the fuel performance code (often taken from the output of a dedicated thermal-hydraulics package). In addition, creep models for the cladding appropriate for this temperature regime are needed. Fission gas release and other fuel-specific models must also be appropriate for these conditions.

Other challenging conditions to analyze, all of which require a multiphysics approach, include reactivity insertion accidents, so-called missing pellet surface defects (a chipped pellet that creates high localized stresses), and stress corrosion cracking of cladding.

Examples of fuel performance codes in use today include TRANSURANUS (Lassmann 1992), ENIGMA (Rossiter 2011), DIONISIO (Soba and Denis 2008), ALCYONE (Michel et al 2013), FRAPCON (Berna et al 1997), FALCON (Rashid et al 2004), and BISON (Williamson et al 2012). BISON is based on the MOOSE framework discussed in Section 3.

Example calculation results for layered 1D (stacked radial slices of fuel and cladding), 2D axisymmetric, and 3D fuel analysis are shown in Figure 1. Low dimensionality models are useful for rapid evaluation of the global fuel rod response, while higher dimensionality models can be used to study local behavior in detail. These models were all run using the BISON code, which uses the same set of computational models with arbitrary dimensionality.
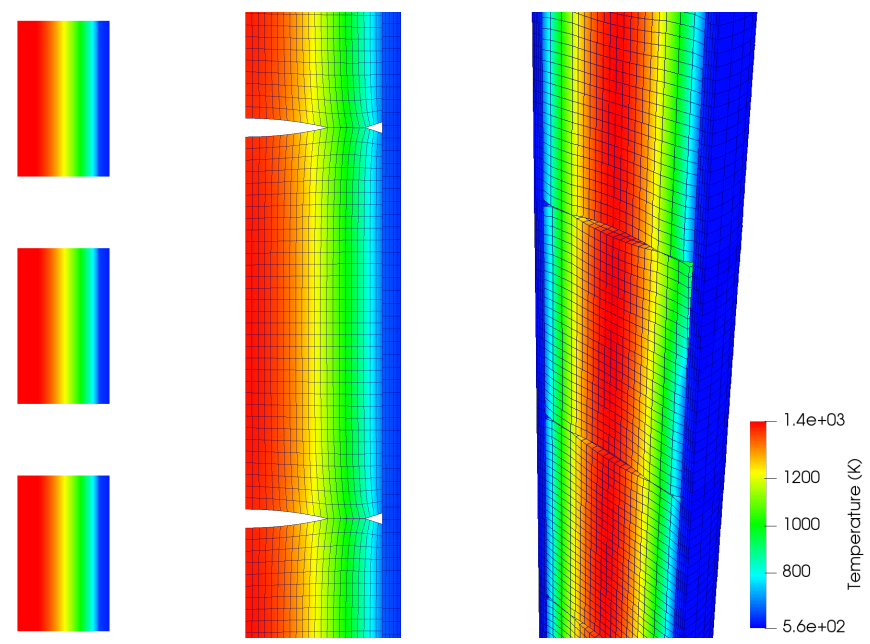

Fig. 1 1D (left), 2D (center), and 3D (right) nuclear fuel models. The 1D model is a set of slices of fuel with radial $1 \mathrm{D}$ elements representing fuel and cladding at a given elevation. The 2D model uses an axisymmetric representation, and can include individual fuel pellets as shown. The 3D model use a symmetry plane through the center of the rod, and can be used to study local effects such as the behavior around a defective pellet on upper right side of center pellet. 


\subsection{Hydride Formation}

Zirconium ( $\mathrm{Zr}$ ) alloys are a well established light water reactor fuel cladding material due to the low neutron capture cross-section of zirconium, its mechanical strength, and good corrosion resistance up to high temperatures.

Fuel elements are in constant contact with the water coolant medium during both reactor operation as well as wet storage, and one of the main challenges facing these alloys is that $\mathrm{Zr}$ has an undesirable reaction with water. At the clad surface, $\mathrm{H}_{2} \mathrm{O}$ molecules are split and zirconium oxides are formed. A portion of the remaining Hydrogen $(\mathrm{H})$ atoms diffuse through the oxide layer forming on that surface into the bulk of the cladding. Continued $\mathrm{H}$ uptake pushes the material past the $\mathrm{H}$ solubility limit, at which point a phase change to a thermodynamically more stable hydride phases is initiated.

Hydride formation has a substantial impact on the engineering scale mechanical properties of the clad material. The low fracture toughness of the $\mathrm{ZrH}$ phases causes clad embrittlement and can lead to clad failure due to fracture.

This solid state diffusion and phase change reaction lends itself to modeling using the phase field method. Required parameterization includes the bulk free energies of the various $\mathrm{ZrH}$ phases, the interfacial free energy between phases, and the kinetic coefficients, i.e. interfacial and species mobilities. Data for this can be found in the literature.

The multiphysics aspects of this problem arise from the fact that the hydride phase transformations result in lattice changes that exhibit volume changes, and thus misfit strains with respect to the $\mathrm{Zr}$ matrix. These strains result in elastic stress fields, which in turn affect the stored mechanical energy of the system. This mechanical energy contributes to the total free energy of the system, the chemical potentials of the diffusing species, and as a result, the microstructure evolution. Further complications can arise from local plastic deformations due to dislocations emitted at points of stress concentrations at developing hydride precipitates.

The mechanism of delayed hydride cracking is a diffusion limited time-dependent crack formation mechanism in which $\mathrm{ZrH}$ platelets are constantly formed at the tip of a crack. The crack propagates in steps through the newly formed hydride as soon as a critical condition, relating to hydride size and stress intensity factors, is met. Inherent to this mechanism is the multiphysics coupling of diffusive species transport, phase transformation, and mechanical state changes.

A comprehensive model for $\mathrm{ZrH}$ formation needs to combine the chemical free energy contributions and the mechanical interactions of the various phases. The resulting coupling in practice is two-fold. One the one hand the evolving phase-field microstructure variables will affect the mechanical properties of the system by means of variable-dependent eigenstrains as well as variable-dependent elasticity tensors. On the other hand the elastic stress state gives rise to an elastic energy $\frac{1}{2} \sigma \epsilon$ which needs to be added to the thermodynamic free energy density used in the phase-field evolution equations. The elastic free energy is a function of a subset of the phase field order parameters though the mechanical properties, which depend on the microstruc- 
tural state. As a consequence, driving forces for these phase field order parameters emerge from the variational derivative of this free energy contribution.

The phase-field and mechanics equations can be solved implicitly in a tightly coupled system using the finite element method. Figure 2 shows a representative coupled phase-field and mechanical finite element simulation of hydride precipitation. In periodic systems under the small strain assumption spectral methods can solve this system effectively as well.

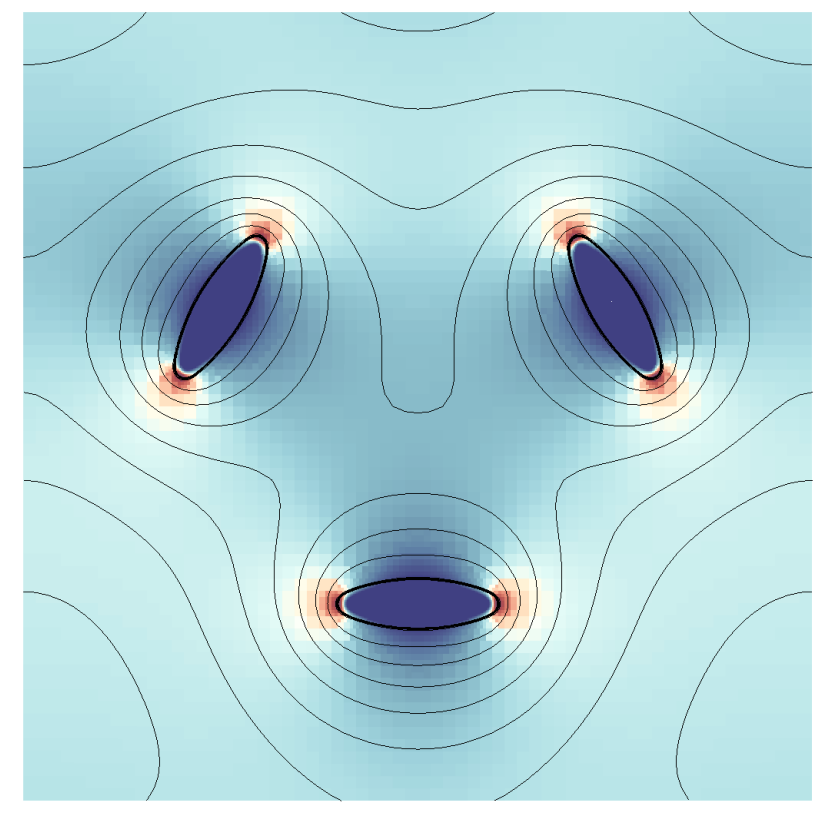

Fig. 2 Stress field and concentration contours of three differently oriented $\gamma$ hydride precipitates.

\section{3 $\mathrm{UO}_{2}$ Microstructure Evolution}

Uranium dioxide $\left(\mathrm{UO}_{2}\right)$, a common light water reactor fuel, is a polycrystalline ceramic material. For reactor applications, the fuel is synthesized in powder form and pressed into cylindrical pellets with a diameter and height of about a centimeter. These pellets are then sintered under a reducing atmosphere and loaded into zirconium alloy cladding tubes. Under operation conditions in a critical nuclear reactor, fission of the ${ }^{235} \mathrm{U}$ nuclei generates fission fragments, neutrons (to keep up the fission reaction), prompt $\gamma$-rays, and - through decaying fission products - $\beta^{-}$-particles, delayed $\gamma$-rays, and heat due to neutron capture. As a consequence the nuclear fuel heats up, incurs lattice damage from collision cascades, and is doped with a broad 
variety of new chemical species - the fission fragments. About $80 \%$ of the heat is deposited during the stopping process of the fission fragments, and roughly $15 \%$ of the fission products deposited in the fuel are insoluble gases.

These harsh conditions result in driving forces that strongly impact the microstructural evolution of the fuel. The techniques described in Section 4.1 are necessary to compute the temperature and stress conditions in the fuel. Thermal and stress gradients affect the migration of both chemical species as well as irradiation generated point defects and defect clusters in the fuel. This requires solving a diffusional or phase field problem.

Beyond these basic mechanisms, which already form a connected multiphysics problem, are the advanced mechanisms of irradiation effects, added chemical species, creep, and fracture. The constant internal irradiation generates a defect population far above the thermal equilibrium. Defects interact with each other to form complex defect structures such as loops or voids and they interact with solute species such as fission gas atoms to form trap sites or gas bubbles. The irradiation also forces chemical mixing through ballistic displacements of atoms leading, for example, to re-solution of fission gas out of the precipitated bubbles. Chemical species intentionally added to the fuel affect its composition and must be taken into account. Figure 3 shows a simulation of the evolution of fission gas bubbles at grain boundaries.

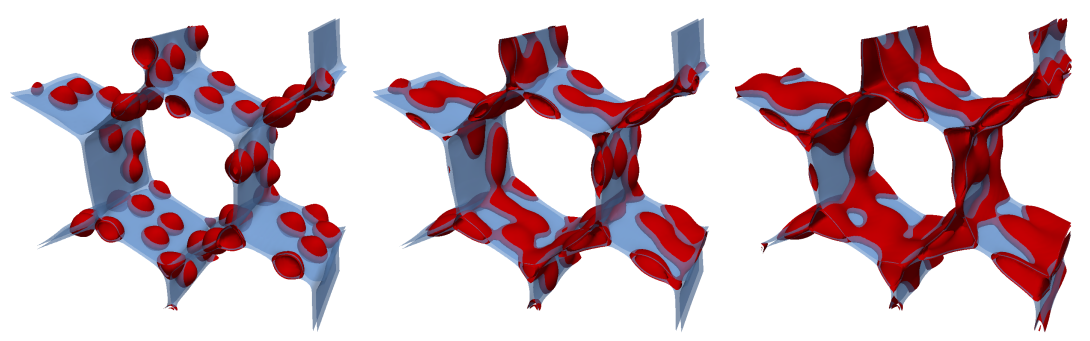

Fig. 3 3D simulation of increasing fission gas grain boundary coverage with progressing burnup in $\mathrm{UO}_{2}$ (from Aagesen et al (2017)).

At the mesoscale level, radiation effects can be simulated with varying levels of fidelity. If the primary concern is the non-equilibrium defect population, phase field simulations can include source terms for the vacancy and interstitial fields. These source terms can be homogeneous, to represent mean field defect production, empirical distributions fitted to typical collision cascade dimensions, or data obtained from a coupled damage calculation code. The most established code for ion transport in matter and the generation of point defects is the binary collision Monte Carlo (BCMC) code TRIM/SRIM (Ziegler et al 2010), which is based on a scattering integral approximation of the Ziegler-Biersack-Littmark potential. BCMC data can also be used to introduce ballistic mixing contributions to a phase field microstructure evolution simulation. 
At the grain level, recrystallization can occur in nuclear fuel as the existing grains acquire a free energy contribution from stored defect populations, such as dislocations. In absence of a fast mechanism to relieve the grains of the stored defects by annihilation or diffusion into sinks, new, defect-free grains with a lower free energy may spontaneously nucleate and form small grained microstructures. This process can be captured by phase field simulations.

\subsection{Summary}

Many aspects of the behavior of components and materials in the nuclear reactor environment are fundamentally affected by phenomena involving the coupled response of multiple physical systems. Including this coupled multiphysics behavior in simulation models is essential for accurately representing many problems of engineering significance.

This chapter provides an overview of the main governing equations involved, the techniques for discretization and solution of these equations, and the software algorithms, frameworks, and codes used for solving multiphysics problems. It also provides an overview of important aspects of a few areas of interest in multiphysics simulation of nuclear reactor components and materials. This is a broad and constantly developing field. The algorithms, codes, and applications described here are not intended to be a comprehensive listing, but are representative of the current state of the art.

Acknowledgements This manuscript has been authored by Battelle Energy Alliance, LLC under Contract No. DE-AC07-05ID14517 with the U.S. Department of Energy. The United States Government retains and the publisher, by accepting the article for publication, acknowledges that the United States Government retains a nonexclusive, paid-up, irrevocable, world-wide license to publish or reproduce the published form of this manuscript, or allow others to do so, for United States Government purposes.

\section{References}

Aagesen L, Schwen D, Zhang Y (2017) Microstructure-level modeling of stage 3 fission gas release in $\mathrm{UO}_{2}$ fuel. Tech. Rep. INL/EXT-17-43374 Rev. 0, Idaho National Laboratory

Allen SM, Cahn JW (1972) Ground state structures in ordered binary alloys with second neighbor interactions. Acta Metallurgica 20(3):423-433, DOI 10.1016/0001-6160(72)90037-5

Bažant ZP, Chern JC, Thonguthai W (1981) Finite element program for moisture and heat transfer in heated concrete. Nuclear Engineering and Design 68(1):61-70, DOI 10.1016/0029-5493(82) 90040-1

Berna GA, Beyer GA, Davis KL, Lanning DD (1997) FRAPCON-3: A computer code for the calculation of steady-state, thermal-mechanical behavior of oxide fuel rods for high burnup. Tech. Rep. NUREG/CR-6534-Vol.2; PNNL-11513-Vol.2, Nuclear Regulatory Commission, Washington, DC 
Cahn JW, Hilliard JE (1958) Free Energy of a Nonuniform System. I. Interfacial Free Energy. The Journal of Chemical Physics 28(2):258-267, DOI 10.1063/1.1744102

Chen L, Shen J (1998) Applications of semi-implicit Fourier-spectral method to phase field equations. Computer Physics Communications 108(2-3):147-158, DOI 10.1016/S0010-4655(97) 00115-X

COMSOL, Inc (2017) COMSOL Multiphysics Reference Manual, version 5.3. URL www. comsol.com

Fick A (1855) On liquid diffusion. Poggendorffs Annalen 94:59, DOI 10.1016/0376-7388(94) 00230-V, reprinted in "On liquid diffusion". Journal of Membrane Science. 100:33-38, 1995.

Fink JK (2000) Thermophysical properties of uranium dioxide. J Nuclear Materials 279(1):1-18

Gaston D, Newman C, Hansen G, Lebrun-Grandié D (2009) MOOSE: A parallel computational framework for coupled systems of nonlinear equations. Nuclear Engineering and Design 239(10):1768-1778, DOI 10.1016/j.nucengdes.2009.05.021

Knoll D, Keyes D (2004) Jacobian-free Newton-Krylov methods: a survey of approaches and applications. Journal of Computational Physics 193(2):357-397, DOI 10.1016/j.jcp.2003.08.010

Lassmann K (1992) TRANSURANUS: a fuel rod analysis code ready for use 188:295-302

Liu R, Prudil A, Zhou W, Chan PK (2016) Multiphysics coupled modeling of light water reactor fuel performance. Progress in Nuclear Energy 91:38-48, DOI 10.1016/j.pnucene.2016.03.030

Logg A, Mardal KA, Wells G (eds) (2012) Automated Solution of Differential Equations by the Finite Element Method, Lecture Notes in Computational Science and Engineering, vol 84. Springer Berlin Heidelberg, Berlin, Heidelberg, DOI 10.1007/978-3-642-23099-8

Lucuta PG, Matzke HJ, Hastings IJ (1996) A pragmatic approach to modelling thermal conductivity of irradiated $\mathrm{UO}_{2}$ fuel: review and recommendations. J Nuclear Materials 232:166-180

Michel B, Nonon C, Sercombe J, Michel F, Marelle V (2013) Simulation of pellet-cladding interaction with the PLEIADES fuel performance software environment. Nuclear Technology 182(2):124-137, DOI 10.13182/NT13-A16424

Novascone S, Spencer B, Hales J, Williamson R (2015) Evaluation of coupling approaches for thermomechanical simulations. Nuclear Engineering and Design 295:910-921, DOI 10.1016/j. nucengdes.2015.07.005

Olander D (1976) Fundamental aspects of nuclear reactor fuel elements. Tech. Rep. TID-26711P1, Technical Information Center, Office of Public Affairs, Energy Research and Development Administration, DOI 10.2172/7343826

Oterkus S, Madenci E (2017) Peridynamic modeling of fuel pellet cracking. Engineering Fracture Mechanics DOI 10.1016/j.engfracmech.2017.02.014

Pawlowski RP, Phipps ET, Salinger AG (2012a) Automating embedded analysis capabilities and managing software complexity in multiphysics simulation, Part I: Template-based generic programming. Scientific Programming (2):197-219

Pawlowski RP, Phipps ET, Salinger AG, Owen SJ, Siefert CM, Staten ML (2012b) Automating embedded analysis capabilities and managing software complexity in multiphysics simulation, Part II: Application to partial differential equations. Scientific Programming (3):327-345, DOI 10.3233/SPR-2012-0351

Prud'homme C (2006) A domain specific embedded language in $\mathrm{C}++$ for automatic differentiation, projection, integration and variational formulations. Scientific Programming 14(2):81110, DOI $10.1155 / 2006 / 150736$

Rahman M, Saghir M (2014) Thermodiffusion or Soret effect: Historical review. International Journal of Heat and Mass Transfer 73:693-705, DOI 10.1016/j.ijheatmasstransfer.2014.02.057

Rashid MM (1993) Incremental kinematics for finite element applications. International Journal for Numerical Methods in Engineering 36(23):3937-3956, DOI 10.1002/nme.1620362302

Rashid Y, Dunham R, Montgomery R (2004) Fuel analysis and licensing code: FALCON MOD01 - Volume 1: Theoretical and numerical bases. Tech. Rep. EPRI-1011307

Rossiter G (2011) Development of the ENIGMA fuel performance code for whole core analysis and dry storage assessments. Nuclear Engineering and Technology 43(6):489-498

Saad Y, Schultz MH (1986) GMRES: a generalized minimal residual algorithm for solving linear systems. SIAM J Sci Statist Comput 7:856 
Silling S (2000) Reformulation of elasticity theory for discontinuities and long-range forces. Journal of the Mechanics and Physics of Solids 48(1):175-209, DOI 10.1016/S0022-5096(99)00029-0

Simo JC, Hughes TJR (1998) Computational inelasticity. No. v. 7 in Interdisciplinary applied mathematics, Springer, New York

Soba A, Denis A (2008) Simulation with DIONISIO 1.0 of thermal and mechanical pellet-cladding interaction in nuclear fuel rods. Journal of Nuclear Materials 374(1):32 - 43, DOI https://doi. org/10.1016/j.jnucmat.2007.06.020

Stafford D (2015) Multidimensional simulations of hydrides during fuel rod lifecycle. Journal of Nuclear Materials 466:362-372, DOI 10.1016/j.jnucmat.2015.06.037

Stewart JR, Edwards H (2004) A framework approach for developing parallel adaptive multiphysics applications. Finite Elements in Analysis and Design 40(12):1599-1617, DOI 10.1016/j.finel. 2003.10.006

Systemes D (2016) Abaqus Unified FEA User Documentation. URL www . 3ds . com

Williamson RL, Hales JD, Novascone SR, Tonks MR, Gaston DR, Permann CJ, Andrs D, Martineau RC (2012) Multidimensional multiphysics simulation of nuclear fuel behavior. J Nuclear Materials 423:149-163, DOI 10.1016/j.jnucmat.2012.01.012

Ziegler JF, Ziegler M, Biersack J (2010) SRIM - The stopping and range of ions in matter (2010). Nuclear Instruments and Methods in Physics Research Section B: Beam Interactions with Materials and Atoms 268(11-12):1818-1823, DOI 10.1016/j.nimb.2010.02.091

Zienkiewicz OC, Taylor RL, Zhu JZ (2013) The finite element method: its basis and fundamentals, seventh edn. Elsevier, Butterworth-Heinemann, Amsterdam

Zinkle S, Was G (2013) Materials challenges in nuclear energy. Acta Materialia 61(3):735-758, DOI 10.1016/j.actamat.2012.11.004 